\title{
Ingestibilité et digestibilité chez le mouton des foins de cinq graminées tropicales
}

\author{
C. Kaboré-Zoungrana ${ }^{1}$ A. Toguyeni ${ }^{1}$ Y. Sana ${ }^{1}$
}

\begin{abstract}
Mots-clés
O vin Djallonké - Andropogon gayanus Panicum anabaptistum - Pennisetum pedicellatum - Brachiaria lata - Andropogon pseudapricus - Prise alimentaire Digestibilité - Valeur nutritive - Burkina Faso.
\end{abstract}

\begin{abstract}
Résumé
Cinq graminées fourragères tropicales, deux pérennes (Andropogon gayanus Kunth. et Panicum anabaptistum Steud.) et trois annuelles (Pennisetum pedicellatum Trin., Brachiaria lata Schum. et Andropogon pseudapricus Stapf.), ont été exploitées à divers stades phénologiques se situant entre les stades tallage et paille. Les quantités de matière sèche (MS) volontairement ingérées par le mouton Djallonké de 23 foins de ces graminées ont varié de 108 à $26 \mathrm{~g} / \mathrm{kg}$ de poids métabolique, selon l'espèce et le stade phénologique. Ces foins se sont caractérisés par des teneurs en matières azotées totales (MAT) faibles (131 à $8 \mathrm{~g} / \mathrm{kg} \mathrm{MS}$ ). Les teneurs en matières azotées digestibles (MAD) des rations composées de ces foins, distribués seuls ou complémentés avec des sources azotées (tourteau de coton, fanes de légumineuses), étaient positivement et étroitement liées ( $r=0,996$; écart type résiduel $(E T R)=2,61 ; n=36)$ aux teneurs en MAT ingérées. La diminution de la digestibilité de la matière organique ( $\mathrm{dMO}$ ) au cours du cycle $(0,21$ point/jour) a entraîné une diminution d'ingestion de $1,6 \mathrm{~g}$ de matière sèche par kilogramme de poids métabolique et par point de $\mathrm{dMO}$. Cette $\mathrm{dMO}$ a été très dépendante des teneurs en parois non digérées (NDFnd) de ces foins ( $r=0,979$; $E T R=2,15 ; n=23$ ), et la fraction soluble S (1 000 - NDF) a eu une digestibilité réelle de l'ordre de 100 p. 100.
\end{abstract}

\section{INTRODUCTION}

Un des problèmes importants auquel est confronté l'élevage des pays soudano-sahéliens est la fluctuation considérable avec la saison de l'état corporel et du poids des animaux. Le système d'élevage traditionnel des ruminants, totalement tributaire du pâturage naturel et des résidus de culture, vit au rythme des saisons et de la pluviométrie. Chaque année, les longues transhumances effectuées par le bétail sont de moins en moins efficaces, l'espace pastoral se rétrécissant sans cesse.

En attendant l'essor de la culture fourragère, qui est une pratique naissante au Burkina Faso et confinée aux zones aménagées, la constitution de réserves fourragères à partir des productions naturelles semble incontournable si l'on veut couvrir ne serait-ce que les besoins d'entretien des animaux et/ou obtenir une légère production durant la longue saison sèche. Partant de ce constat, l'apport d'aliments complémentaires sous forme de foins a été

1. IDR, Université polytechnique de Bobo-Dioulasso, 01 BP 1091, BoboDioulasso 01, Burkina Faso envisagé dans cette étude. Encore faut-il alors s'assurer que la valeur alimentaire (ingestibilité et digestibilité) de ces derniers est en mesure de pallier à un tel déficit quantitatif et qualitatif des ressources fourragères.

Des valeurs de digestibilité d'espèces tropicales (dont Andropogon gayanus et Pennisetum pedicellatum) existent dans la littérature. Il ressort, par exemple, des nombreuses données compilées par Butterworth et Butterworth (4), une forte variabilité liée à l'espèce, mais les stades phénologiques et les conditions de mesures ne sont pas toujours précisés, alors qu'ils sont d'importants facteurs de variation.

Il est par conséquent nécessaire pour les espèces végétales étudiées ici que des valeurs nutritives soient données pour des stades phénologiques définis. De plus, la comparaison entre ces espèces sera d'autant plus valable que les mesures auront été effectuées dans les mêmes conditions.

Cinq graminées fourragères parmi les plus représentatives de la zone Nord soudanienne du Burkina Faso ont été retenues. Il s'agit de deux espèces pérennes, Andropogon gayanus Kunth. et Panicum anabaptistum Steud., et de trois espèces annuelles, Pennisetum pedicellatum Trin., Andropogon pseudapricus Stapf. et Brachiaria lata Schum. 


\section{MATERIEL ET METHODES}

Les espèces étudiées ont été choisies pour leur appétibilité et leur contribution spécifique élevée sur les pâturages de la zone d'étude à la Station expérimentale de Gampéla $\left(12^{\circ} 25^{\prime}\right.$ 'de latitude Nord et $1^{\circ} 21^{\prime}$ de longitude Ouest) où s'est déroulée l'étude. Ces espèces se retrouvent sur trois types de pâturages : pâturages de plateau, pâturages de bas-fond et pâturages intermédiaires entre le plateau et le bas-fond. Elles y forment par endroits de larges plages continues quasi monospécifiques.

Les graminées ont été exploitées à divers stades phénologiques se situant entre les stades tallage et paille (tableau I). Le fourrage une fois fauché a été préfané au soleil une journée ou deux (en fonction du temps), puis séché à l'ombre sous un hangar, avec plusieurs retournements.

Le foin ainsi préparé a été distribué ad libitum à des moutons adultes mâles castrés de race Djallonké et d'un poids vif moyen de 21,67 $\pm 1,18 \mathrm{~kg}$. Le taux de refus toléré avec les fourrages tempérés (10 p. 100) est difficilement transposable aux fourrages tropicaux, du fait de leur caractère plus grossier, et a donc été majoré (15 à 25 p. 100). Cependant, la limite supérieure de 25 p. 100 était difficile à respecter pour certains stades, selon l'espèce, sous peine de mettre en péril la vie des animaux. Pour ces raisons, les taux de refus ont été élevés à 40 p. 100 pour les stades paille de Pennisetum pedicellatum et maturation d'Andropogon gayanus et à 30 p. 100 pour cette dernière espèce à partir de l'épiaison.

Certains foins tardifs ont été non seulement distribués seuls mais aussi parfois avec un complément azoté. Dans ce cas, les valeurs obtenues n'ont été utilisées que pour le calcul des liaisons dMA/MAT.

Pour chaque mesure d'ingestibilité et de digestibilité, un lot homogène de six animaux a été constitué sur la base du poids vif. Ces moutons ont été maintenus en cages à métabolisme individuelles, ce qui a permis la mesure exacte des quantités d'aliments offertes et refusées ainsi que des fèces excrétées. La période pré-expérimentale de 15 jours a été suivie d'une période de collecte des fèces de sept jours.

La composition chimique des fourrages distribués et refusés et celle des fèces ont été déterminées. Les analyses chimiques suivantes ont été effectuées : matière sèche (MS) à l'étuve à $105^{\circ} \mathrm{C}$ pendant $24 \mathrm{~h} ;$ cendres totales $(\mathrm{MM})$ par calcination de la matière sèche à $550^{\circ} \mathrm{C}$; matières azotées totales (MAT) correspondant à l'azote selon Kjeldalh ( $\mathrm{N}$ x 6,25); constituants pariétaux, NDF (neutral detergent fiber), ADF (acid detergent fiber) dosé directement sur l'échantillon et ADL (acid detergent lignin) sulfurique déterminé à partir de l'ADF ; énergie par calorimétrie.

\section{Tableau I}

Q uantité de matière sèche ingérée $\left(\mathrm{g} / \mathrm{kg} \mathrm{p}^{0,75}\right)$ en fonction du stade phénologique

\begin{tabular}{|c|c|c|c|c|c|c|}
\hline \multirow{2}{*}{$\begin{array}{l}\text { Stade } \\
\text { phénologique }\end{array}$} & & \multicolumn{2}{|c|}{ Pérennes } & \multicolumn{3}{|c|}{ Annuelles } \\
\hline & & Ag & $\mathrm{Pa}$ & Ap & BI & $\mathrm{Pp}$ \\
\hline $\begin{array}{l}\text { Tallage } \\
\text { (début) }\end{array}$ & $\begin{array}{l}\text { MSVI }\left(g / k g p^{0,75}\right) \\
\text { dMO }(\%) \\
\text { Taux de refus }(\%)\end{array}$ & $\begin{array}{l}68 \\
56 \\
22\end{array}$ & & & & $\begin{array}{l}74 \\
66 \\
17\end{array}$ \\
\hline $\begin{array}{l}\text { Tallage } \\
\text { (fin) }\end{array}$ & $\begin{array}{l}\text { MSVI }\left(g / k g p^{0,75}\right) \\
\text { dMO (\%) } \\
\text { Taux de refus (\%) }\end{array}$ & $\begin{array}{l}53 \\
49 \\
23\end{array}$ & $\begin{array}{l}57 \\
51 \\
32\end{array}$ & & & $\begin{array}{l}81 \\
67 \\
20\end{array}$ \\
\hline Montaison & $\begin{array}{l}\text { MSVI }\left(g / k g p^{0,75}\right) \\
\text { dMO }(\%) \\
\text { Taux de refus }(\%)\end{array}$ & $\begin{array}{l}40 \\
43 \\
31\end{array}$ & & $\begin{array}{l}45 \\
53 \\
22\end{array}$ & $\begin{array}{c}108 \\
68 \\
12\end{array}$ & $\begin{array}{l}72 \\
61 \\
17\end{array}$ \\
\hline Epiaison & $\begin{array}{l}\left.\text { MSVI (g/kg } \mathrm{p}^{0,75}\right) \\
\text { dMO }(\%) \\
\text { Taux de refus }(\%)\end{array}$ & $\begin{array}{l}32 \\
43 \\
36\end{array}$ & $\begin{array}{l}58 \\
45 \\
21\end{array}$ & $\begin{array}{l}41 \\
48 \\
25\end{array}$ & $\begin{array}{l}98 \\
61 \\
22\end{array}$ & $\begin{array}{l}60 \\
58 \\
27\end{array}$ \\
\hline Fructification & $\begin{array}{l}\left.\text { MSVI ( } / / k g p^{0,75}\right) \\
\text { dMO }(\%) \\
\text { Taux de refus }(\%)\end{array}$ & $\begin{array}{l}38 \\
39 \\
31\end{array}$ & $\begin{array}{l}53 \\
43 \\
24\end{array}$ & & & $\begin{array}{l}50 \\
58 \\
20\end{array}$ \\
\hline Maturation & $\begin{array}{l}\left.\text { MSVI (g/kg } \mathrm{p}^{0,75}\right) \\
\text { dMO }(\%) \\
\text { Taux de refus }(\%)\end{array}$ & $\begin{array}{l}26 \\
31 \\
43\end{array}$ & & & & $\begin{array}{l}42 \\
48 \\
28\end{array}$ \\
\hline D issémination & $\begin{array}{l}\left.\text { MSVI (g/kg } \mathrm{p}^{0,75}\right) \\
\text { dMO (\%) } \\
\text { Taux de refus (\%) }\end{array}$ & & $\begin{array}{l}50 \\
32 \\
21\end{array}$ & & & \\
\hline Paille & $\begin{array}{l}\left.\text { MSVI (g/kg } \mathrm{p}^{0,75}\right) \\
\text { dMO }(\%) \\
\text { Taux de refus }(\%)\end{array}$ & & $\begin{array}{l}30 \\
39 \\
33\end{array}$ & $\begin{array}{l}31 \\
46 \\
27\end{array}$ & & $\begin{array}{l}34 \\
38 \\
40\end{array}$ \\
\hline
\end{tabular}

Andropogon gayanus (Ag), Panicum anabaptistum (Pa), Andropogon pseudapricus (Ap), Brachiaria lata (B1), Pennisetum pedicellatum (Pp) MSVI : matière sèche volontairement ingérée ; $\mathrm{dMO}$ : digestibilité de la matière organique 
La valeur nutritive des foins est déterminée en calculant leurs valeurs énergétiques et azotées. Les valeurs énergétiques sont exprimées en unités fourragères lait (UFL), calculées à partir de l'énergie digestible (1) ; les valeurs azotées, exprimées en protéines digestibles dans l'intestin (PDI), sont calculées à partir des équations proposées par l'Inra (13), intégrant les teneurs en matières azotées totales et en cellulose brute du fourrage.

Les résultats obtenus ont été analysés au moyen du système Statgraphics (21). L'étude des relations entre les différentes variables a été effectuée par une analyse de régression et de variance (ANOVA). Les différences considérées comme significatives le sont au seuil de probabilité de $p<0,05$.

\section{RESU LTATS}

\section{Composition chimique des fourrages}

Le tableau II donne, par espèce, les valeurs extrêmes des teneurs en constituants chimiques des fourrages distribués. Ces teneurs ont varié selon l'espèce et le stade. A stade phénologique équivalent, les teneurs en MAT ont été supérieures chez Brachiaria lata, puis chez Pennisetum pedicellatum, Panicum anabaptistum, Andropogon gayanus et Andropogon pseudapricus. Les teneurs en parois totales (NDF), à l'inverse, ont été plus faibles.

La diminution des teneurs en MAT avec l'âge a été très importante au cours du cycle. Elle a été plus brutale en début de végétation pour les espèces annuelles et plus faible par la suite pour toutes les espèces entre la montaison et les stades reproducteurs.

\section{Ingestibilité}

Les quantités de matière sèche volontairement ingérées par le mouton ont varié de 1081 à $268 \mathrm{~g}$, soit de 108 à $26 \mathrm{~g} / \mathrm{kg}$ de poids métabolique $\left(\mathrm{p}^{0,75}\right)$ selon l'espèce et le stade phénologique (tableau I).
Brachiaria lata a été l'espèce consommée en plus grande quantité A l'épiaison, son ingestibilité a été de $98 \mathrm{~g} / \mathrm{kg} \mathrm{p}^{0,75}$, supérieure à celle de toutes les autres espèces, même lorsqu'elles étaient au stade très jeune. Cependant, les différences d'ingestibilité liées à l'espèce ont été plus faibles que celles liées aux stades phénologiques, c'est-à-dire à l'âge des espèces.

Sur des durées de mesure variant, selon l'espèce, de 30 à 95 jours la diminution journalière a été voisine $\left(0,39\right.$ à $\left.0,49 \mathrm{~g} / \mathrm{kg} \mathrm{p}^{0,75}\right)$, bien que, pour une espèce donnée, elle n'ait pas été uniforme tout au long du cycle et qu'elle ait eu tendance à s'accroître avec l'âge du fourrage.

\section{Digestibilité}

La digestibilité des 23 foins de graminées a varié de 67 à 30 p. 100 pour la matière sèche (dMS) et de 68 à 31 p. 100 pour la matière organique $(\mathrm{dMO})$ selon l'espèce et le stade phénologique (figure 1). A stade phénologique équivalent, les espèces ont différé du point de vue de la dMS et de la dMO :

Brachiaria lata $>$ Pennisetum pedicellatum $>$ Andropogon pseudapricus $>$ Panicum anabaptistum $=$ Andropogon gayanus

A un stade donné, la plus grande différence de dMO entre espèces (Brachiaria lata et Andropogon gayanus à la montaison) a atteint 25 points et la plus faible (Andropogon pseudapricus et Andropogon gayanus au stade paille) 8 points.

La diminution journalière de la $\mathrm{dMO}$ des foins * a été en moyenne de 0,21 point. La diminution au cours du cycle (stade tallage à paille) a été considérable. Elle a été de 18 points chez Andropogon gayanus.

La digestibilité des parois (dNDF et dADF) a varié respectivement de 77 à 32 p. 100 et de 69 à 26 p. 100. La digestibilité de la cellulose brute $(\mathrm{dCB})$ a varié de 70 à 40 p. 100 et a été en moyenne supérieure de 4 points à la dADF (tableau II).

* Sauf pour Brachiaria lata, non évaluée à cause du nombre insuffisant de données

\section{Tableau II}

Valeurs extrêmes des teneurs en constituants chimiques (g/kg M S), de la digestibilité (\%) des parois et de la valeur nutritive des foins de graminées exprimée en unités fourragères lait (UFL), protéines digestibles dans I'intestin (PDI) et en matière organique digestible ingérée (MODI)

\begin{tabular}{|c|c|c|c|c|c|}
\hline & $\mathrm{Pp}$ & Ag & $\mathrm{Pa}$ & Ap & BI \\
\hline \multicolumn{6}{|c|}{ Constituants chimiques } \\
\hline Cendres & 138-81 & $63-40$ & $63-50$ & $70-47$ & $121-120$ \\
\hline MAT & $96-16$ & $57-18$ & $70-17$ & $25-8$ & $131-120$ \\
\hline NDF & $644-803$ & $748-828$ & $755-848$ & $794-819$ & $649-686$ \\
\hline ADF & $353-520$ & $396-520$ & $399-446$ & $494-538$ & $313-327$ \\
\hline$A D L$ & $23-69$ & $29-61$ & $47-65$ & $28-63$ & $33-41$ \\
\hline $\mathrm{CB}$ & $331-474$ & $370-492$ & $382-447$ & $464-506$ & $305-313$ \\
\hline \multicolumn{6}{|c|}{ Digestibilité des parois } \\
\hline NDF & $72-45$ & $63-32$ & $58-35$ & $55-47$ & $73-61$ \\
\hline ADF & $69-40$ & $59-30$ & $52-26$ & $56-52$ & $65-54$ \\
\hline CB & $70-43$ & $62-42$ & $51-42$ & $63-55$ & $75-62$ \\
\hline \multicolumn{6}{|l|}{ Valeur nutritive } \\
\hline UFL & $0,59-0,16$ & $0,41-0,08$ & $0,39-0,13$ & $0,24-0,19$ & $0,84-0,62$ \\
\hline PDI & 48-6 & $29-5$ & $28-5$ & 8-7 & 90-81 \\
\hline MODI (g/kg p $\left.{ }^{0,75}\right)$ & $46-12$ & $33-13$ & $28-9$ & $23-14$ & $64-52$ \\
\hline
\end{tabular}

Pennisetum pedicellatum (Pp), Andropogon gayanus (Ag), Panicum anabaptistum (Pa), Andropogon pseudapricus (Ap), Brachiaria lata (Bl) 

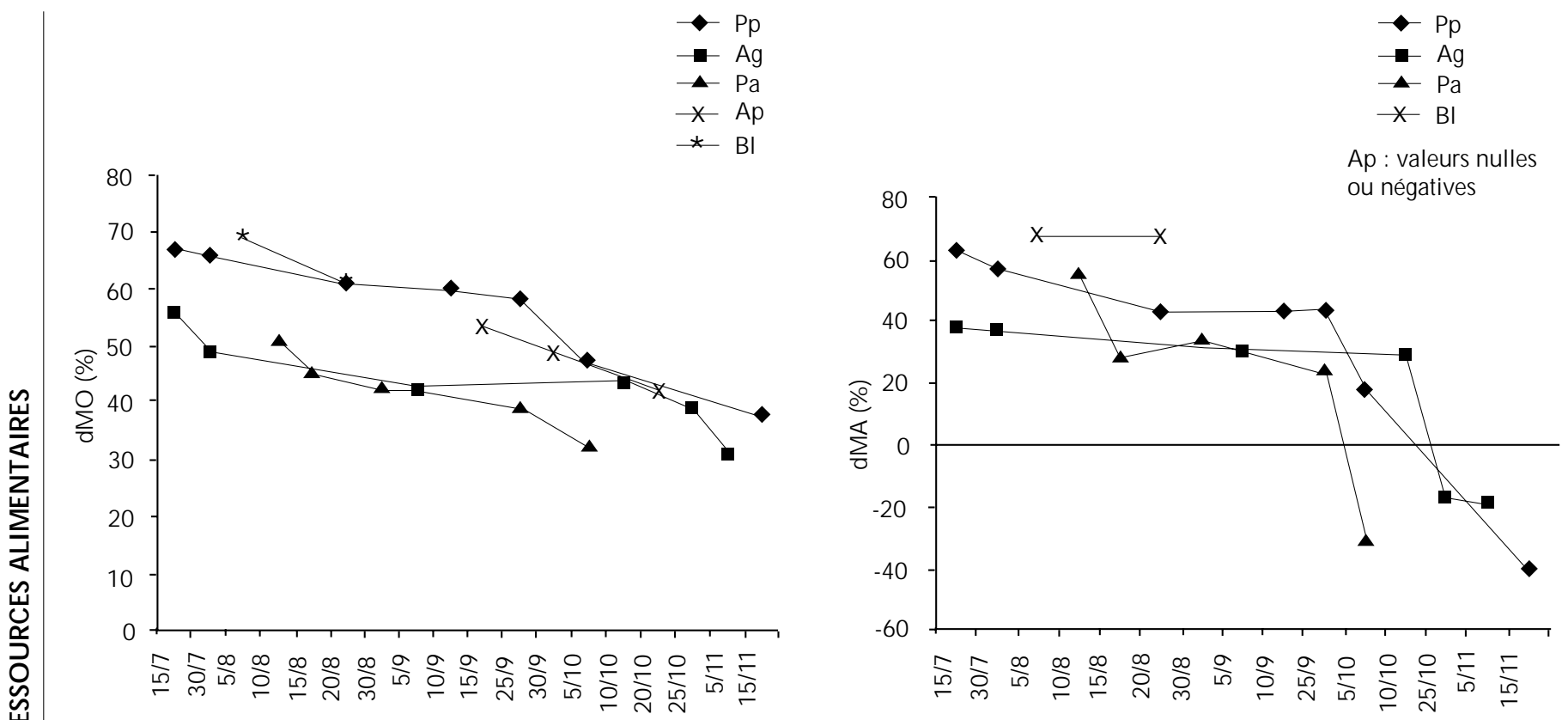

Figure 1 : évolution au cours du cycle de végétation de la digestibilité $(\%)$, de la matière organique (dMO) et des matières azotées (dM A) de Pennisetum pedicellatum (Pp), Andropogon gayanus (Ag), Panicum anabaptistum (Pa), Andropogon pseudapricus (Ap) et Brachiaria lata (BI).

La digestibilité des matières azotées (dMA) des foins a été beaucoup plus variable que leur dMO (figure 1). La dMA maximale a été de 68 p. 100 (Brachiaria lata à la montaison) et les dMA ont été nulles et négatives respectivement à partir de la montaison pour Andropogon pseudapricus et du stade paille pour toutes les autres espèces.

\section{Relation entre l'ingestibilité et la digestibilité}

L'exploitation plus tardive du fourrage s'est traduite par une baisse des quantités de foin ingérées par l'animal, suite à une modification de la composition chimique et de la digestibilité de la matière organique. Une diminution d'un point de la dMO s'est traduite par une diminution d'ingestion de $1,6 \mathrm{~g}$ de $\mathrm{MS} / \mathrm{kg} \mathrm{p}^{0,75}$ (figure 2).

L'espèce végétale a cependant été un facteur de variation important, puisque, avec la même digestibilité, la quantité de MS ingérée pouvait être très différente. C'est ainsi que, par exemple, avec Brachiaria lata au stade montaison elle a été de $36 \mathrm{~g} / \mathrm{kg} \mathrm{p}^{0,75}$ (soit de 49 p. 100) plus élevée qu'avec Pennisetum pedicellatum au même stade. Cette différence correspond à $21 \mathrm{~g}$ de matière organique digestible ingérée (MODI) par kilogramme de poids métabolique, soit l'équivalent du besoin d'entretien.

Les teneurs en MAT du foin distribué expliquent au mieux $(\mathrm{r}=+0,89)$ la variation de la quantité de MS ingérée (figure 3$)$. Les liaisons avec les éléments des parois ont été moins étroites : $r=-0,84$ pour l'ADF $; r=-0,77$ pour le NDF $; r=-0,31$ pour l'ADL.

\section{Relation entre les teneurs en MAD et MAT}

A partir des foins distribués seuls et de rations composées de ces mêmes foins, complémentés avec des sources de matières azotées (fanes de légumineuses ; tourteaux de coton, $\mathrm{n}=13$ ), une relation linéaire avec une précision acceptable (écart type résiduel (ETR) $=2,67)$ a pu être établie entre les teneurs en MAD et MAT des foins (figure 4).

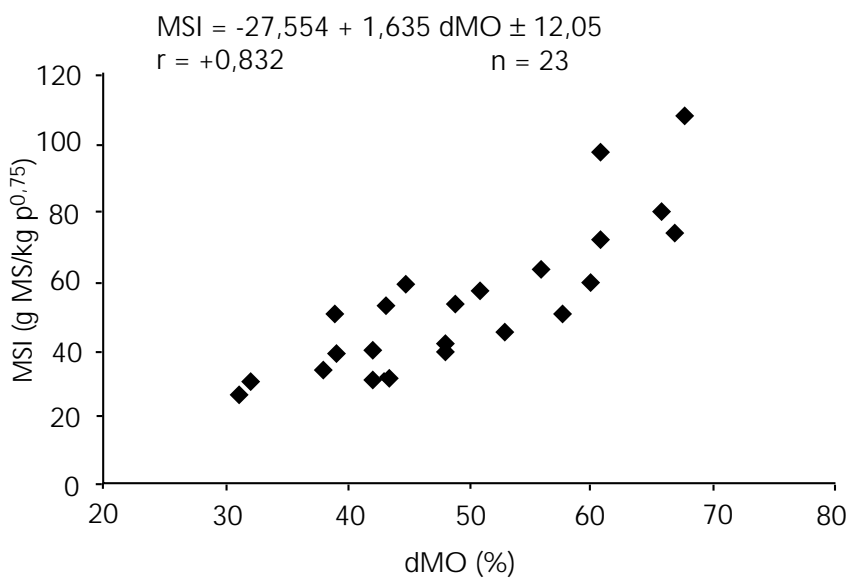

Figure 2 : variation de l'ingestibilité (MSI) en fonction de la digestibilité de la matière organique ( $\mathrm{dMO})$ des foins.

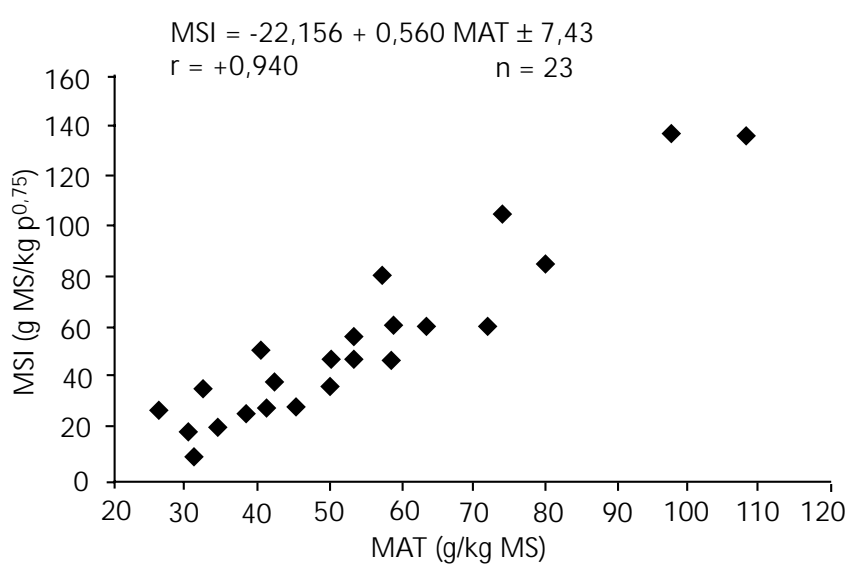

Figure 3 : variation de l'ingestibilité (MSI) en fonction des matières azotées totales (MAT) des foins. 


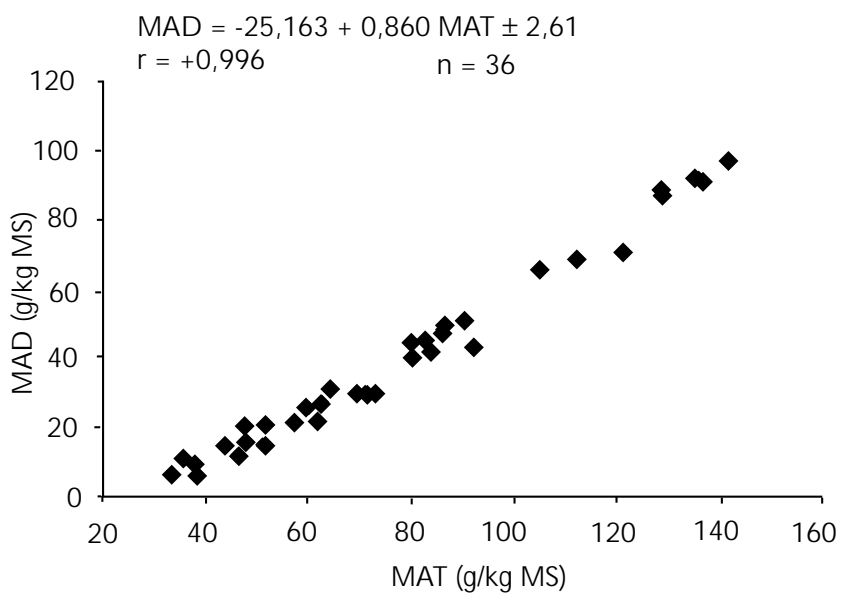

Figure 4 : relation entre les teneurs en matières azotées digestibles (MAD) et les teneurs en matières azotées totales (MAT) des foins seuls ou complémentés.

La digestibilité réelle des MAT a été très élevée (86 p. 100). Les teneurs en matières azotées non digestibles (MAND) calculées à partir des teneurs en MAT et de la digestibilité apparente ont été de l'ordre de $35,5 \pm 5,3 \mathrm{~g}$.

\section{Relation entre la digestibilité des parois et les teneurs en ces parois}

Des différents constituants des parois (NDF, ADF et ADL), seule la teneur en NDF a été liée significativement à la dNDF $(r=-0,57)$. L'écart type résiduel élevé (11 points de digestibilité) s'explique par le fait qu'à teneur équivalente de NDF dans l'ingéré, la digestibilité pouvait être très différente selon l'espèce. Elle a été par exemple de 65 et 40 p. 100 respectivement chez Pennisetum pedicellatum et Andropogon gayanus pour une teneur dans l'ingéré de $750 \mathrm{~g}$. La différence doit provenir, du moins en partie, des teneurs en ADL plus faibles pour Pennisetum pedicellatum que pour Andropogon gayanus (41 contre $57 \mathrm{~g} / \mathrm{kg} \mathrm{MS}$ ).

En revanche, la fraction soluble $(\mathrm{S})$ des foins correspondant à celle éliminée lors du dosage du NDF a été totalement utilisable, comme en témoigne la relation linéaire établie entre la digestibilité de cette fraction (SD) et les teneurs (figure 5). La digestibilité réelle de $\mathrm{S}$ a été de l'ordre de 100 p. 100.

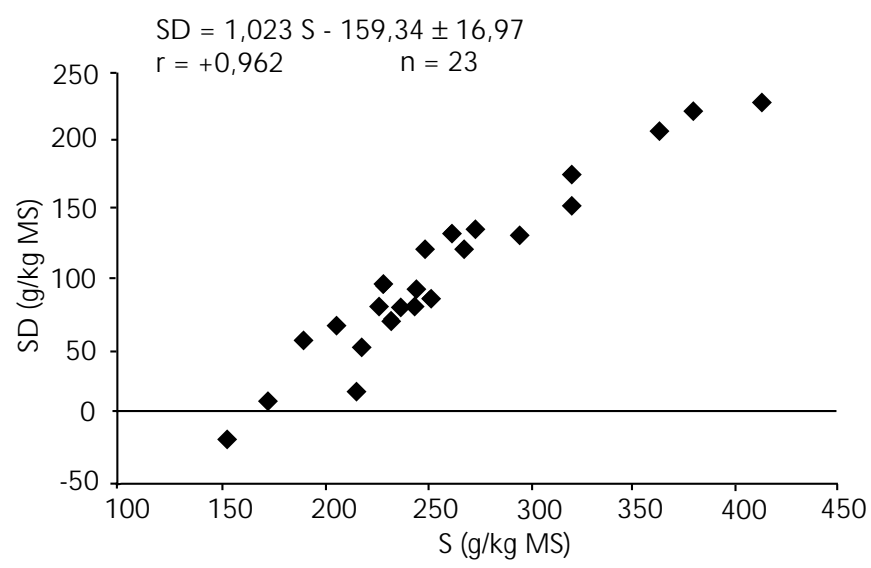

Figure 5 : relation entre les fractions soluble digestible (SD) et soluble ingérée (S) des foins de graminées.

\section{DISCUSSIO N}

De nombreuses valeurs d'ingestibilité de fourrages tropicaux existent dans la littérature pour les espèces distribuées en vert $(9,17$, 19) ou sous forme de foins (de 35 à $\left.68,5 \mathrm{~g} / \mathrm{kg} \mathrm{p}^{0,75}\right)(5,11,22)$. Des taux de refus variables (de 5 à plus de 50 p. 100) ont été tolérés, alors que l'ingestibilité a été influencée par les quantités de MS offertes (12) qui conditionnent le degré de tri opéré par l'animal (23) dans le cas des fourrages pauvres.

Des cinq espèces étudiées, Brachiaria lata était la plus digestible, contenait plus de constituants solubles digestibles et moins de parois non digestibles et avait aussi l'ingestibilité la plus élevée.

L'ingestibilité et la dMO des foins étudiés ont évolué dans le même sens. La théorie de la limitation de l'ingestibilité par la masse de parois dans le rumen, et surtout sa dépendance vis-à-vis de la vitesse de dégradation après un temps de séjour court (8), est largement reconnue, faisant du NDF un critère de prévision relativement efficace pour la prédiction de l'ingestibilité (10). Cependant, c'est la teneur en MAT des foins qui permet de prédire au mieux l'ingestibilité, grâce, probablement, à une plage de variation des teneurs en MAT importante et aussi parce que la teneur en MAT évolue, comme la proportion de feuilles dont l'ingestibilité est bien supérieure à celle des tiges. Mais cela peut aussi être dû en partie au fait que des teneurs faibles en MAT n'assurent pas les besoins de la microflore et inhibent ainsi la cellulolyse (15).

Des évolutions et des valeurs de dMO comparables à celles obtenues dans cette étude ont été observées pour des foins de graminées tropicales par d'autres auteurs : 70 à 54 p. 100 (22) et 59 p. 100 (11). La diminution moyenne de la dMO, comprise entre 0,19 et 0,23 point par jour, était comparable à celle trouvée pour les fourrages verts tropicaux, mais moins variable : 0,21 à 0,39 point/jour $(6,7)$ ou 0,18 à 0,41 point/jour $(20)$.

La dMO était positivement et étroitement corrélée à la digestibilité des parois totales et, plus que les teneurs dans l'aliment, elle était très dépendante des teneurs en fraction non digérée des parois (NDFnd) (figure 6). Ces résultats concordent avec ceux d'Aufrère et coll. (2). Il existe en fait peu de constituants intracellulaires d'origine alimentaire dans les fèces, comme le confirme l'équation établie pour les foins de cette étude entre la fraction soluble et sa digestibilité, ou pour d'autres fourrages tropicaux $(5,18)$. La fraction indigestible de $159 \mathrm{~g}$ de fraction soluble par kilogramme de matière sèche ingérée serait donc essentiellement d'origine

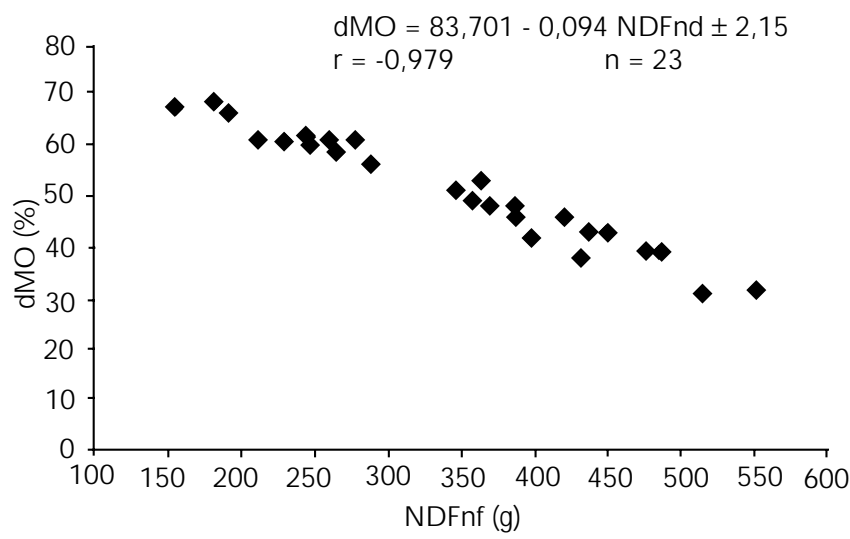

Figure 6 : relation entre la digestibilité de la matière organique ( $\mathrm{dMO}$ ) et les teneurs en NDF (neutral detergent fiber) non digestible (NDFnd) des foins de graminées. 
métabolique. La fraction de NDFnd évolue comme la teneur en NDF des foins ; elle s'accroît avec l'âge du fourrage, conséquence $\mathrm{du}$ vieillissement, de la lignification et de la modification de la composition morphologique.

L'évolution au cours du cycle de végétation de la dMA, plus importante que celle de la dMO, a suivi celle de la teneur en MAT des fourrages. La digestibilité réelle des MAT des foins (86 p. 100) a été identique à celle trouvée pour un nombre plus important de foins tropicaux (16), mais elle a été légèrement plus faible que celle obtenue pour des fourrages verts tropicaux $(6,20)$.

La dMA apparente n'a guère dépassé 68 p. 100, à cause des teneurs élevées en azote des fèces provenant, d'une part, de la fraction alimentaire non digestible (en moyenne 14 p. 100), qui correspondrait en majorité à la fraction d'azote liée aux parois, et, surtout, des $25 \mathrm{~g}$ de MAT d'origine non alimentaire, excrétés par kilogramme de matière sèche ingérée, constitués à 57-87 p. 100 par des résidus bactériens (14).

\section{- CONCLUSION}

Les valeurs d'ingestibilité et de digestibilité, en dehors de Brachiaria lata et des stades jeunes des autres espèces, ont été plutôt faibles. Elles ont conduit à une production animale limitée par un apport azoté ou énergétique déficient. La couverture du besoin d'entretien (estimé à 0,44 UFL et 34 g PDI) ne peut être assurée par les seules distributions de Pennisetum pedicellatum au stade tallage et de Brachiaria lata jusqu'à l'épiaison.

On sait que les valeurs d'ingestibilité et de digestibilité des foins sont plus faibles que celle des fourrages verts correspondants. Il n'empêche que la production animale a été généralement faible, voire négative, sur les pâturages de la zone d'étude, au delà de l'épiaison. Le facteur incriminé le plus limitant était sans doute l'ingestion de MS qui a été plus variable (de 1,9 fois) que la digestibilité. Elle conditionne, plus que cette dernière, la quantité de MOD ingérée par les animaux.

La capacité d'ingestion limitée a probablement résulté des teneurs élevées en parois des foins, entraînant une vitesse de dégradation plus lente, associée à un apport d'azote insuffisant pour l'activité cellulolytique et la couverture des besoins des animaux.

Le contenu des espèces au stade retenu pour leur exploitation, moins de parois et plus de MODI, pourrait servir de critères de choix et de classification. Dans ce cas, Brachiaria lata est de loin la meilleure.

\section{BIBLIO GRAPH IE}

1. ANDRIEU J., DEMARQ UILLY C., WEGAT-LITRE E., WEISS Ph., 1981. Prévision de la valeur énergétique des foins. In : Prévision de la valeur nutritive des aliments des ruminants. Versailles, France, Inra publications, p. 119-127.

2. AUFRERE J., GRAVIOU D., DEMARQUILLY C., ANDRIEU J., EMILE J.C., GIOVAN NI R., MAUPETIT P., 1992. Estimation of organic matter digestibility of whole maize plants by laboratory methods. Anim. Feed Sci. Technol., 36: 187-204.

3. BUTTERWORTH M.H., 1967. The digestibility of tropical grasses. N utr. Abstr. Rev., 37: 349-351.

4. BUTTERW ORTH M.H., BUTTERW ORTH J., 1965. Some aspects of utilisation of tropical forages. 2. Pangola and costal Bermuda hays. J. Agric. Sci., 65: 389-395.

5. CARLIER L.A., COTTYN B.J., AERT J.V., 1976. Apparent and true digestibility of Weende components, cell content and cell wall of ryegrass. An. Feed Sci. Technol., 1: 607-617.

6. CHENOST M., 1973. La valeur alimentaire de quatre graminées et d'une légumineuse tropicales et ses facteurs de variation. Fourrages, $\mathbf{5 4}$ 87-108.

7. CHENOST M., 1975. La valeur alimentaire du Pangola (Digitaria decumbens stent.) et ses facteurs de variation, en zone tropicale humide. Ann. Zootech., 24 : 327-349.

8. DEMARQUILLY C., CHEN OST M., 1969. Etude de la digestion des fourrages dans le rumen par la méthode des sachets de nylon; liaison avec la valeur alimentaire. Ann. Zootech., 18 : 419-436.

9. DIEN G A., BULDGEN A., COMPERE R., 1991. La culture fourragère temporaire d'Andropogon gayanus Kunth var. bisquamulatus en zone soudano-sahélienne sénégalaise - 4 . Composition chimique et valeur alimentaire du fourrage. Bull. Rech. agro. Gembloux, 26 : 351-366.

10. DULPHY J.P., 1987. Prévision de l'ingestibilité des foins chez le mouton à partir de leur teneur en parois. Reprod. Nutr. Dévelop., 27 193-194.
11. GIHAD E.A., 1976. Intake, digestibility and nitrogen utilisation of tropical natural grass hay by goat and sheep. J. Anim. Sci., 43: 879-883.

12. HAGGAR R.J., AHMED M.B., 1970. Seasonal production of Andropogon gayanus - II. Seasonal changes in digestibility and feed intake. J. agric. Sci., 77: 47-52.

13. INRA, 1978. Alimentation des ruminants. Versailles, France, Inra publications, $597 \mathrm{p}$.

14. MASON V.C., 1969. Some observations on the distribution and origin of nitrogen in sheep faeces. J. agric. Sci., 73: 99-111.

15. MILFORD R., MINSON D.J., 1965. Intake of tropical pasture species. In: Proc. IXth International Grassland Congress, Sao Paulo, Brazil, 07-20 January 1965, p. 815-822.

16. MILFORD R., MINSON D.J., 1965. The relation between the crude protein content and digestible crude protein content of tropical pasture plants. J. Br. Grassl. Soc., 20: 177-179.

17. MINSON D.J., 1972. The digestibility and voluntary intake by sheep of six tropical grasses. Aust. J. exp. Agric. Anim. Husb., 12: 21-27.

18. MIN SON D.J., 1984. Digestibility and voluntary intake by sheep of five Digitaria species. Aust. J. exp. Agric. Anim. Husb., 24: 494-500.

19. MINSON D.J., BRAY R.A., 1986. Voluntary intake and in vivo digestibility by sheep of five lines of Cenchrus ciliaris selected on the basis of preference rating. Grass Forage Sci., 41: 47-52.

20. RICHARD D., 1987. Valeur alimentaire de quatre graminées fourragères tropicales. Maisons-Alfort, France, Cirad-lemvt, $314 \mathrm{p}$.

21. STATGRAPHICS, 1988. Statistical graphics system. Cary, NC, USA, STSC.

22. VENTURA M., MOORE J.E., RUELKE O.C., FRANKE D.E., 1975. Effect of maturity and protein supplementation on voluntary intake and nutrient digestibility of Pangola digitaria grass hays. J. Anim. Sci., 40: 769-774.

23. ZEMMELINK G., HAGGAR R.J., DAVIES J.H., 1972. A note on voluntary intake of Andropogon gayanus hay by cattle, as affected by level of feeding. Anim. Prod., 15: 85-88.

Reçu le 5.9.97, accepté le 10.9.99 


\section{Summary}

Kaboré-Zoungrana C., Toguyeni A., Sana Y. Feed intake and digestibility by sheep of five tropical grass hays

Five tropical grass hays, two perennial (Andropogon gayanus Kunth. and Panicum anabaptistum Steud.) and three annual (Pennisetum pedicellatum Trin., Brachiaria lata Schum. and Andropogon pseudapricus Stapf.) were used at various phenological stages occurring between tillering and the straw stage. Depending on the species and phenological stage, voluntary intake amounts of dry matter (DM) of 23 grass hays by Djallonke ( $=$ W est African D warf) sheep varied from 108 to $26 \mathrm{~g} / \mathrm{kg}$ of metabolic weight. Total crude protein contents (TCP) of these hays were low (131 to $8 \mathrm{~g} / \mathrm{kg} D M)$. Digestible crude protein (DCP) contents of the hay rations, given alone or protein-supplemented (with cottonseed cake, legume haulms) were positively and closely related $(r=0.996$; residual standard error (RSE) $=2.61 ; n=36$ ) to ingested TCP contents. Decrease of organic matter digestibility $(\mathrm{OMd})$ during the cycle $(0.21$ point/day) induced an intake decrease of $1.6 \mathrm{~g} \mathrm{DM}$ per kilogram of metabolic weight and per $\mathrm{OMd}$ point. O Md was highly correlated to the non-digested wall contents (NDFnd) of the hays $(r=0.979 ; \operatorname{RSE}=2.15 ; n=23)$, and $S$ the soluble fraction (1000 - NDF) had a true digestibility of about $100 \%$.

Key words: Djallonke sheep - Andropogon gayanus Panicum anabaptistum - Pennisetum pedicellatum Brachiaria lata - Andropogon pseudapricus - Feed intake Digestibility - Nutritive value - Burkina Faso.

\section{Resumen}

Kaboré-Zoungrana C., Toguyeni A., Sana Y. Ingestión y digestibilidad en el cordero de los henos de cinco gramíneas tropicales

Cinco gramíneas forrajeras tropicales, dos perennes (Andropogon gayanus Kunth. y Panicum anabaptistum Steud.) y tres anuales (Pennisetum pedicellatum Trin., Brachiaria lata Schum. y Andropogon pseudapricus Staf.), fueron explotadas a diversos estadios fenológicos, entre el estadio de macolla y el de paja. Las cantidades de materia seca ingeridas voluntariamente por el cordero Djallonké de 23 henos de estas gramíneas variaron de 108 a $26 \mathrm{~g} / \mathrm{kg}$ de peso metabólico, según la especie y el estadio fenológico. Estos henos se caracterizaron por tenencias en materias nitrogenadas totales (MAT) bajas (131 a $8 \mathrm{~g} / \mathrm{kg} \mathrm{MS}$ ). Las tenencias en materias nitrogenadas digeribles (MAD) de las raciones compuestas de estos henos, distribuidas solas o suplementadas con fuentes nitrogenadas (torta de algodón, hojas secas de leguminosas), estuvieron positiva y estrechamente ligadas $(r=0,996$; desviación estándar residual $(E T R)=2,61 ; n=36$ ) a las tenencias en MAT ingerida. La disminución de la digestibilidad de la materia orgánica (dM O) durante el ciclo (0,21 punto/día) indujo a una disminución de la ingestión de 1,6 g de materia seca por kilogramo de peso metabólico y por punto de dMO. Esta dMO fue altamente dependiente de las tenencias en las paredes no digeridas (N DFnd) de estos henos ( $r=0,979$; ETR $=2,75 ; n=23)$ y la fracción soluble $\mathrm{S}(1000-\mathrm{NDF})$ tubo una digestibilidad real del orden de $100 \%$.

Palabras clave: Ovino Djallonké - Andropogon gayanus Panicum anabaptistum - Pennisetum pedicellatum - Brachiaria lata - Andropogon pseudapricus - Ingestión de piensos Digestibilidad - Valor nutritivo - Burkina Faso. 